\title{
Chylous Ascites Secondary to Giant Liver Hemangioma
}

\author{
Darius L. Lazarus ${ }^{a}$ Said A. Al-Busafi ${ }^{c} \quad$ Nir Hilzenrat ${ }^{b}$ \\ Departments of ${ }^{\mathrm{a}}$ Medicine and ${ }^{\mathrm{b}} \mathrm{Gastroenterology,} \mathrm{Jewish} \mathrm{General} \mathrm{Hospital,}$ \\ McGill University, Montreal, Que., Canada; 'Department of Medicine, \\ College of Medicine and Health Science, Sultan Qaboos University, Al Khoudh, \\ Sultanate of Oman
}

\section{Key Words}

Chyle $\cdot$ Ascites $\cdot$ Chylous ascites $\cdot$ Hemangioma

\begin{abstract}
Chylous ascites is rare in clinical practice. It is characterized by milky-appearing peritoneal fluid with a triglycerides concentration of $>1.25 \mathrm{mmol} / \mathrm{l}(110 \mathrm{mg} / \mathrm{dl})$. Its pathophysiology is related to a disruption in the normal lymphatic flow. It is more common after trauma (including post surgery), neoplasia or atypical infections such as tuberculosis or filariasis. Other rare medical causes have been reported. The treatment is supportive and focused on correction of the underlying pathology. We report here the first case of chylous ascites caused by giant liver hemangioma and discuss the management of this condition.
\end{abstract}

\section{Introduction}

Ascites is the accumulation of fluid in the peritoneal cavity. Although most commonly due to cirrhosis and severe liver disease, ascites can be caused by a multitude of other conditions such as malignancy, heart failure, infections, pancreatic disease or dialysis. Whenever the etiology is not clear, the first step in the evaluation and management of ascites is diagnostic paracentesis. The ascitic fluid sample should be sent for fluid cell count and differential, ascitic fluid total protein and serum-ascites albumin gradient (SAAG). Depending on the aspect of the fluid (milky, turbid, foamy), triglycerides levels should be also obtained and, if infection is suspected, the fluid should be cultured at the bedside in blood culture bottles prior to initiation of antibiotics.

Chylous ascites is a rare form of ascites resulting from the accumulation of lymph in the peritoneal cavity. The peritoneal fluid has a characteristic milky appearance and has a triglyceride concentration of $>1.25 \mathrm{mmol} / \mathrm{l}(110 \mathrm{mg} / \mathrm{dl})$. It is caused by a disruption in the normal lymphatic flow within the abdominal cavity. Increased 
pressure in the lymphatic system secondary to cirrhosis, obstructive infiltrative malignancy or heart failure/pericarditis can eventually cause leakage of lymph into the peritoneal cavity, which has already a reduced absorptive capacity due to venous stasis generated by the above conditions. Trauma or surgery can cause ruptures in the lymphatic system with leakage directly into the peritoneal cavity. If these lymphoperitoneal fistulas are large enough they cannot close by themselves. Surgical re-intervention is sometimes required although they can be very hard to localize. Infections (mainly tuberculosis and tuberculosis species, or filariasis) are also an important cause, especially in patients with suitable epidemiology. Less commonly reported causes are congenital, inflammatory, heart failure and nephrotic syndrome.

Chylous ascites usually manifests as abdominal distension, weight gain and shortness of breath when causing increased abdominal pressure and flattening of the diaphragm. Depending of the underlying etiology the presentation can be acute as in trauma or post abdominal or thoracic surgery, or more chronic as in cirrhosis or malignancy. The diagnosis can hardly be established just by the clinical presentation, and a diagnostic paracentesis is required.

The treatment of chylous ascites is targeted toward the correction of the underlying pathology. If a clear cause cannot be identified or controlled, dietary restriction and several pharmacologic therapies have been reported successful in different studies. Surgery or interventional procedures such as transjugular intrahepatic portosystemic shunt (TIPS) are also sometimes used in selected cases.

\section{Case Report}

We describe the case of a 65-year-old female retired healthcare worker who presented to the emergency department of our hospital with abdominal distension, abdominal pain and increasing shortness of breath. Her symptoms had been subacute in onset and worsening for the 2 weeks prior to presentation. She gave a history of milk of magnesia used for constipation which in her view precipitated her abdominal distension. She reported anorexia, weakness and $5 \mathrm{lbs}$ weight loss in the past and more recent and abrupt weight gain coinciding with increased abdominal girth. She did not have any history of trauma or recent abdominal surgeries, travels, infectious contacts or underlying liver or kidney diseases. She denied smoking, alcohol or intravenous drug abuse. Her family history was unremarkable.

Her past medical history was remarkable for relapsing-remitting multiple sclerosis (treated with interferon beta-1a and prednisone) and hypertension. She had a remote total hysterectomy and bilateral salpingo-oophorectomy for uterine leiomyoma. A liver hemangioma was incidentally discovered on the occasion of a pre-operative abdominal CT scan, but it was deemed of no surgical significance. Her medication included interferon beta-1a, amlodipine, bisoprolol, ramipril, hydrochlorothiazide, pregabalin, folic acid and ferrous sulphate.

On physical exam cachexia and moderate confusion were noted. General examination did not reveal stigmata of chronic liver disease such as spider angiomas, palmar erythema, cutaneous or scleral icterus, or abdominal collateral circulation. A mild flapping tremor was also noted and she presented bilateral Terry's nails. Terry's nails are characterized by loss of lunula with a whitish 'ground glass' appearance of the nail. This is a nonspecific physical finding that is associated with chronic conditions such as cirrhosis, diabetes mellitus, heart failure, hyperthyroidism, and malnutrition. The patient also had mild peripheral edema. Her abdomen was distended and positive for shifting dullness and fluid wave. A grade 3 out of 3 ascitic abdomen as per International Ascites Club classification was appreciated, but no hepatosplenomegaly. The rest of her systemic examination was normal. 
Initial laboratory investigations revealed creatinine $113 \mu \mathrm{mol} / \mathrm{l}$ (normal 45-95), urea $12 \mathrm{mmol} / \mathrm{l}$ (normal 3-8), normal electrolytes, albumin (43 g/l), bilirubin, coagulogram and transaminases. Alkaline phosphatase was increased to $455 \mathrm{U} / \mathrm{l}$ (normal 35-145) and serum tumor markers returned with normal AFP, CEA and CA19-9. Viral markers showed evidence of previous hepatitis A exposure and no active or previous infection with hepatitis B or C viruses.

Abdominal ultrasound confirmed a significant amount of free intraperitoneal fluid. A diagnostic and therapeutic paracentesis was performed and therapeutic drainage was done. The peritoneal fluid was milky in appearance and separated on standing. SAAG was $35 \mathrm{~g} / \mathrm{l}$, indicating a high SAAG ascites. A high SAAG ascites (values $>11 \mathrm{~g} / \mathrm{l}$ ) is characteristic of portal hypertension. As expected, the fluid triglyceride concentration was high at $3.6 \mathrm{mmol} / \mathrm{l}(>1.25 \mathrm{mmol} / \mathrm{l}$ or $110 \mathrm{mg} / \mathrm{dl})$, which placed it in the chylous ascites range.

An infectious etiology was ruled out by negative signs of infection, low white blood cell count in serum and ascites, negative cultures and Gram stain. Furthermore tuberculosis was excluded with negative AFB stains (Kinyoun and auramine, from concentrated specimen), negative BACTEC mycobacterium culture and negative mycobacterium DNA detection by PCR tests. The cytology analysis of the fluid was negative for malignancy.

An echocardiography was obtained and it was negative for signs of heart failure, valvulopathies, pericardial tamponade or constrictive physiology, which excluded these from the differential. Imaging of the thorax and abdomen by CT scan revealed that the right lobe of the liver was almost completely replaced by a large mass showing peripheral discontinuous nodular and progressive enhancement compatible with a hemangioma (fig. 1). The remaining portions of the liver showed multiple smaller hemangiomas. The liver had a micronodular contour with morphologic changes suggestive of cirrhosis. The right portal vein had been replaced by multiple collaterals while the left portal vein was enlarged. A subsequent MRI of the abdomen with contrast obtained in the following weeks showed that one of the liver lesions had grown by approximately $1 \mathrm{~cm}$ in three dimensions. There was a new rounded peripherally T2-hypointense, centrally T1- and T2-hyperintense area of signal within this enlarged hemangioma, suggesting that the interval enlargement was related to an interval hemorrhage (fig. 2). The intrahepatic inferior vena cava was found to be very small in caliber within the upper liver, near its entry into the right atrium. The right hepatic vein was severely narrowed. An ultrasound-guided liver biopsy was performed, targeting normal tissue, and basically showed no cirrhosis, no malignancy, and extensive hemangioma findings. These findings showed impressive extension of her initial hemangioma and progression toward chronic liver disease and portal hypertension leading to chylous ascites.

The patient was initially managed with regular paracenteses with albumin replacement. She was started on regular octreotide $100 \mu \mathrm{g}$ subcutaneously 3 times a day. Medium-chain triglycerides were avoided due to concerns regarding possible hepatic encephalopathy. Although the ascites continued to improve and required less abdominal paracentesis, the patient's condition started to deteriorate with progressive liver failure. She was referred to a liver transplant center for evaluation but unfortunately died shortly after.

\section{Discussion}

As already mentioned in the introduction, chylous ascites is a rare clinical entity. Its incidence varies from $1 / 20,000$ to $1 / 187,000$ admissions to large tertiary centers $[1,2]$. A recent systematic review of atraumatic chylous ascites done by Steinemann et al. [3] found that the most currently encountered etiologies were lymphatic anomalies (32\%), malignancy (17\%), liver cirrhosis (11\%) and Mycobacterium infection (10\%). Miscellaneous causes were reported in $21 \%$ of cases: cardiac (constrictive pericarditis or left ventricular dysfunction), fibrosing mesenteritis, nephrotic syndrome, collagenosis/vasculitis, gastrointestinal disorders such as intestinal malrotation, volvulus or ascaris. 
To our knowledge this is the first case of chylous ascites caused by massive liver hemangioma. All previously reported causes of chylous ascites have in common a disruption of the normal flow within the lymphatic system. The pathophysiology of chylous ascites in this patient might be similar to that of cirrhosis and owing to lymphatic vessel congestion. The massive hemangioma of this patient occupying almost all the volume of her liver caused for sure a mass effect within the liver. This probably resulted in disruption of lymphatic vessels and spill of lymph into the peritoneal cavity.

Assessment of the etiology of any type of ascites is critical for its management. Treatment of Mycobacterium or filarial infection, correction of the heart failure or revision of the underlying malignancy was reported to control and reverse chylous ascites. Specific recommendations directed toward reducing chyle formation and subsequently formation of the chylous ascites are employed in patients in whom a cause cannot be identified or who do not respond to treatment of the underlying cause.

Several case studies reported success in controlling the ascites with somatostatin and octreotide [4-6]. Somatostatin and its more potent analog octreotide are inhibitors of growth hormone, glucagon, insulin and intestinal hormones. They reduce secretion of fluids by the intestine and pancreas and cause vasoconstriction in the splanchnic blood vessels (decreasing portal hypertension) and likely lymphatic vessels. Our patient received regular octreotide $100 \mu \mathrm{g}$ subcutaneously 3 times a day. She responded slowly to octreotide within 2-3 weeks, progressively requiring less paracenteses for comfort, and after week 5 not requiring paracenteses at all.

Dietary restriction of long-chain triglycerides can also be applied to reduce the production of chyle. A high-protein and low-fat diet with medium-chain triglyceride supplementation [7-9] can successfully achieve this goal without nutritionally depleting the patient. Medium-chain triglycerides are easily absorbed into the intestinal cells and then directly transported to the liver by the portal vein without entering the lymphatic stream. They are found in coconut oil, palm kernel oils, and camphor tree drupes. These provide a source of lipids without increasing the production of lymph. However, despite their beneficial effects, medium-chain triglycerides have a tendency to induce ketogenesis and metabolic acidosis. In patients with advanced liver cirrhosis they can aggravate the encephalopathy or even produce coma [10]. We were not able to try this strategy with our patient due to her limited liver reserve. We did however try bowel rest (fasting) with supportive total parenteral nutrition without any improvement.

Other strategies to control chylous ascites included orlistat [11], surgery [12], TIPS and peritoneovenous shunting in cases of refractory ascites. Use of orlistat was avoided in our patient due to risk of liver failure. As no surgical cause or malignancy were identified, a surgical approach was unfeasible. Peritoneovenous shunts are prone to complications such as infection, disseminated intravascular coagulation, electrolyte imbalance, small bowel obstruction, embolism and occlusion. They are seldom used [13]. TIPS is effective in reducing portal hypertension and risk of variceal bleeding in cirrhosis. TIPS was reported to successfully resolve chylous ascites [14] but can increase the hepatic encephalopathy of cirrhotic patients. TIPS was 
of not benefit for our patient, again giving her poor liver reserve and presence of the giant hemangioma.

In conclusion, chylous ascites is a rare clinical finding cause by a disruption of the normal lymphatic flow. Its management is directed toward managament of the cause, therefore the clinician should be aware of the variety of conditions causing chylous ascites. Octreotide, a nonspecific agent that reduces lymph production and flow, can be helpful. Other strategies have been employed with varying rates of success.

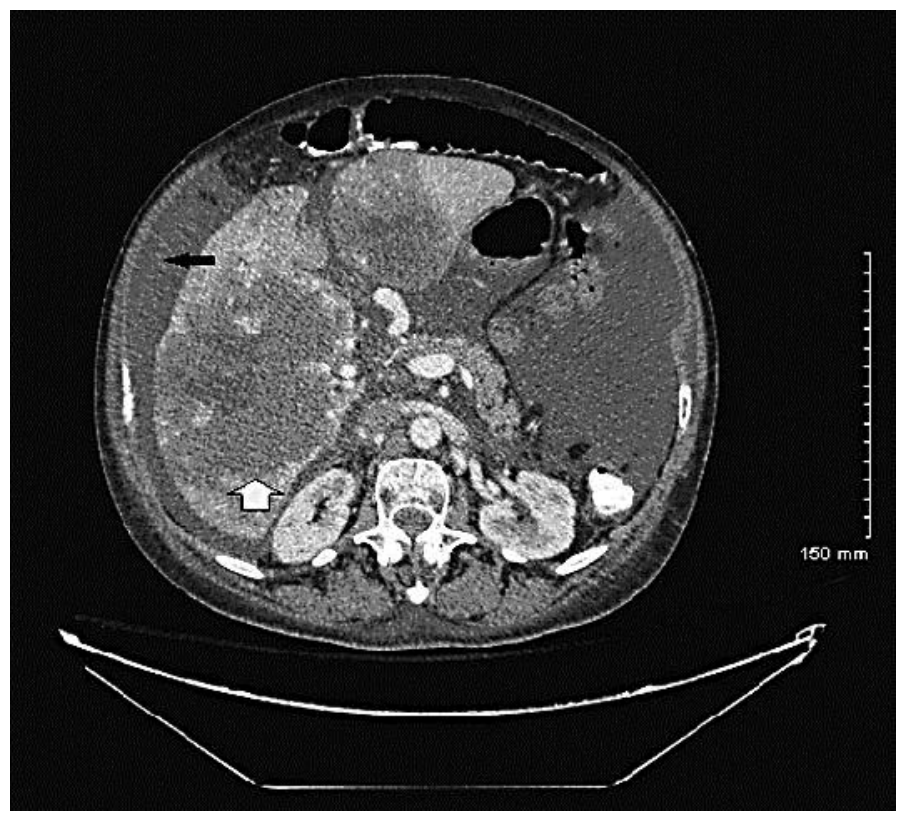

Fig. 1. CT scan slide of the patient. The right lobe of the liver was almost completely replaced by a large mass (white arrow) showing peripheral discontinuous nodular and progressive enhancement compatible with a hemangioma measuring up to $17 \times 15 \times 11 \mathrm{~cm}$. Presence of ascites was also noted (black arrow). 


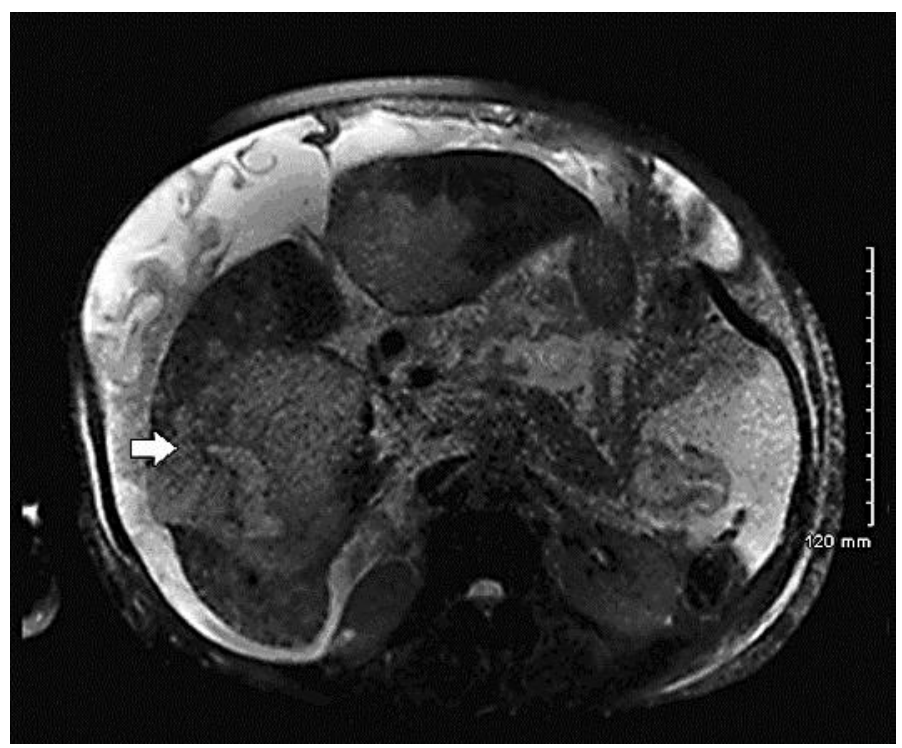

Fig. 2. MRI slide of the patient. Enlarged hemangioma (white arrow).

\section{References}

1 Almakdisi T, Massoud S, Makdisi G: Lymphomas and chylous ascites: review of the literature. Oncologist 2005;10:632-635.

-2 Vasko JS, Tapper RI: The surgical significance of chylous ascites. Arch Surg 1967;95:355-368.

-3 Steinemann DC, Dindo D, Clavien PA, Nocito A: Atraumatic chylous ascites: systematic review on symptoms and causes. J Am Coll Surg 2011;212:899-905.e1-4.

4 Leong RW, House AK, Jeffrey GP: Chylous ascites caused by portal vein thrombosis treated with octreotide. J Gastroenterol Hepatol 2003;18:1211-1213.

5 Mincher L, Evans J, Jenner MW, Varney VA: The successful treatment of chylous effusions in malignant disease with octreotide. Clin Oncol (R Coll Radiol) 2005;17:118-121.

6 Huang Q, Jiang ZW, Jiang J, Li N, Li JS: Chylous ascites: treated with total parenteral nutrition and somatostatin. World J Gastroenterol 2004;10:2588-2591.

-7 Leibovitch I, Mor Y, Golomb J, Ramon J: The diagnosis and management of postoperative chylous ascites. J Urol 2002;167(2 pt 1):449-457.

8 Ohri SK, Patel T, Desa LA, Spencer J: The management of postoperative chylous ascites. A case report and literature review. J Clin Gastroenterol 1990;12:693-697.

-9 Weinstein LD, Scanlon GT, Hersh T: Chylous ascites. Management with medium-chain triglycerides and exacerbation by lymphangiography. Am J Dig Dis 1969;14:500-509.

10 Wieland TM, Lin X, Odle J: Emulsification and fatty-acid chain length affect the utilization of mediumchain triglycerides by neonatal pigs. J Anim Sci 1993;71:1869-1874.

$\checkmark 11$ Chen J, Lin RK, Hassanein T: Use of orlistat (xenical) to treat chylous ascites. J Clin Gastroenterol 2005;39:831-833.

12 Aalami O0, Allen DB, Organ CH Jr: Chylous ascites: a collective review. Surgery 2000;128:761-778.

13 Ablan CJ, Littooy FN, Freeark RJ: Postoperative chylous ascites: diagnosis and treatment. A series report and literature review. Arch Surg 1990;125:270-273.

14 de Vries GJ, Ryan BM, de Bièvre M, Driessen A, Stockbrugger RW, Koek GH: Cirrhosis related chylous ascites successfully treated with TIPS. Eur J Gastroenterol Hepatol 2005;17:463-466. 